\title{
openheart Prospective arrhythmia surveillance after a COVID-19 diagnosis
}

Thomas A Dewland (D) , ${ }^{1}$ Isaac R Whitman, ${ }^{2}$ Sithu Win, ${ }^{3}$ Jose M Sanchez, ${ }^{4}$ Jeffrey E Olgin, ${ }^{5}$ Mark J Pletcher, ${ }^{6}$ Lekshmi Santhosh, ${ }^{5}$ Uday Kumar, ${ }^{7}$ Sean Joyce, ${ }^{5}$ Vivian Yang, ${ }^{5}$ Janet Hwang, ${ }^{5}$ Kelsey Ogomori, ${ }^{5}$ Noah Peyser, ${ }^{5}$ Cathy Horner, ${ }^{5}$ David Wen (D) , ${ }^{5}$ Xochitl Butcher, ${ }^{5}$ Gregory M Marcus ${ }^{1}$

To cite: Dewland TA, Whitman IR, Win S, et al. Prospective arrhythmia surveillance after a COVID-19 diagnosis. Open Heart 2022;9:e001758. doi:10.1136/ openhrt-2021-001758

Received 22 June 2021 Accepted 31 December 2021

Check for updates

(c) Author(s) (or their employer(s)) 2022. Re-use permitted under CC BY-NC. No commercial re-use. See rights and permissions. Published by BMJ.

${ }^{1}$ Department of Medicine, University of California San Francisco, San Francisco, California, USA

${ }^{2}$ Department of Medicine, Temple University, Philadelphia, Pennsylvania, USA

${ }^{3}$ Department of Medicine, ZSFGH, San Francisco,

California, USA

${ }^{4}$ Department of Medicine, University of Colorado, Denver, Colorado, USA

${ }^{5}$ Department of Medicine, UCSF, San Francisco, California, USA ${ }^{6}$ Department of Epidemiology and Biostatistics, UCSF, San Francisco, California, USA ${ }^{7}$ Element Science, Inc, San Francisco, California, USA

Correspondence to Dr Gregory M Marcus; greg. marcus@ucsf.edu

\section{ABSTRACT}

Background Cardiac arrhythmias have been observed among patients hospitalised with acute COVID-19 infection, and palpitations remain a common symptom among the much larger outpatient population of COVID-19 survivors in the convalescent stage of the disease. Objective To determine arrhythmia prevalence among outpatients after a COVID-19 diagnosis.

Methods Adults with a positive COVID-19 test and without a history of arrhythmia were prospectively evaluated with 14-day ambulatory electrocardiographic monitoring. Participants were instructed to trigger the monitor for palpitations.

Results A total of 51 individuals (mean age $42 \pm 11$ years, $65 \%$ women) underwent monitoring at a median 75 (IQR 34-126) days after a positive COVID-19 test. Median monitoring duration was 13.2 (IQR 10.5-13.8) days. No participant demonstrated atrial fibrillation, atrial flutter, sustained supraventricular tachycardia (SVT), sustained ventricular tachycardia or infranodal atrioventricular block. Nearly all participants (96\%) had an ectopic burden of $<1 \%$; one participant had a $2.8 \%$ supraventricular ectopic burden and one had a $15.4 \%$ ventricular ectopic burden. While 47 (92\%) participants triggered their monitor for palpitation symptoms, $78 \%$ of these triggers were for either sinus rhythm or sinus tachycardia.

Conclusions We did not find evidence of malignant or sustained arrhythmias in outpatients after a positive COVID-19 diagnosis. While palpitations were common, symptoms frequently corresponded to sinus rhythm/sinus tachycardia or non-malignant arrhythmias such as isolated ectopy or non-sustained SVT. While these findings cannot exclude the possibility of serious arrhythmias in select individuals, they do not support a strong or widespread proarrhythmic effect of COVID-19 infection after resolution of acute illness.

\section{INTRODUCTION}

Although pneumonia is the most common manifestation of COVID-19 infection, a subset of patients will also develop significant cardiovascular complications. Reports have detailed a variety of cardiac pathologies associated with COVID-19, including systolic heart failure, myocarditis and acute coronary syndrome. ${ }^{1} \mathrm{It}$ is hypothesised that COVID-19 can result in

\section{Key questions}

What is already known about this subject?

- Both atrial and ventricular arrhythmias have been described among patients hospitalised with acute COVID-19 infection. While palpitations remain a frequent symptom among the ambulatory population of COVID-19 survivors in the convalescent stage of the disease, the correlation between these symptoms and identifiable rhythm disturbances is not well studied.

What does this study add?

- In this prospective observational cohort study that enrolled ambulatory individuals with a recent and confirmed COVID-19 infection, we found a very low incidence of clinically significant cardiac arrhythmias using continuous 14-day electrocardiographic monitoring.

How might this impact on clinical practice?

- Although these findings cannot exclude the possibility of serious arrhythmia in select individuals, they do not support a strong or widespread proarrhythmic effect of COVID-19 infection after resolution of acute illness.

myocardial injury through several pathways, including direct infection of myocardial cells, vascular inflammation, thromboembolism and virus-associated cytokine release. ${ }^{2}$ Hospitalised patients with evidence of myocardial damage suffer a markedly increased risk of inpatient mortality. ${ }^{34}$

Both atrial and ventricular arrhythmias have also been described among patients hospitalised with acute COVID-19 infection. ${ }^{4-8}$ As these inpatients are a highly select cohort of individuals most severely affected by the disease, it is likely that indirect factors, including hypoxia, myocardial ischemia, systemic inflammation, haemodynamic instability, pharmacologic therapy and electrolyte disturbances, confound the association between infection and arrhythmia. It therefore may not be appropriate to extrapolate 
findings observed among the most severely ill to the much more commonly seen outpatient with COVID-19. While palpitations remain a frequent symptom among the ambulatory population of COVID-19 survivors in the convalescent stage of the disease, ${ }^{9}{ }^{10}$ the correlation between these symptoms and identifiable rhythm disturbances is not well studied.

We therefore used 14-day ambulatory electrocardiographic monitoring to determine the prevalence of cardiac arrhythmias among outpatients after a COVID-19 diagnosis. By enrolling non-hospitalised individuals, we sought to assess the residual risk of arrhythmia after recovery from acute infection while minimising the proarrhythmic bias introduced by critical illness.

\section{METHODS}

Individuals $\geq 18$ years of age without a history of arrhythmia were invited to participate in the study after a positive COVID-19 test. Recruitment efforts included (1) contacting consecutive individuals with a positive test performed at the University of California San Francisco (UCSF) and (2) inviting participation among those enrolled in the UCSF COVID-19 Citizen Science Study (CCS). Briefly, the UCSF COVID-19 CCS is an international, internet-based cohort study with over 49000 remotely enrolled participants designed to study COVID-19 epidemiology and risk factors. Participants diagnosed with COVID-19 outside of UCSF were required to provide documentation of their positive test result. Individuals with a self-reported history of a cardiac arrhythmia diagnosed prior to COVID-19 infection or who were receiving therapy with a beta blocker, calcium channel blocker or Vaughan-Williams Class I or Class III antiarrhythmic drug prior to infection were excluded. Study candidates with active palpitation symptoms after their COVID-19 diagnosis were neither encouraged nor discouraged from enrolment. Individuals previously hospitalised for COVID-19 were eligible for enrolment after hospital discharge. Pregnant women were excluded.

A remote, video-assisted intake visit was used to obtain demographic information, medical history and current medications. Individuals who reported cigarette, cigar, marijuana, electronic nicotine or any other similar inhaled/vaped product within the 30 days prior to screening were classified as active smokers. Peak COVID-19 symptoms were classified as mild if they did not interfere with normal daily activities, moderate if there was some limitation and severe if there was significant functional limitation. On enrolment, participants were mailed a Zio XT monitor (iRhythm Technologies, San Francisco, California). Monitor application was supervised by the study team via a second video visit. Participants were instructed to wear the monitor for 14 days. No specific activity instructions or physical limitations were provided. Participants were asked to trigger the monitor if they experienced palpitations. After 14 days, the monitor was returned to iRhythm via mail and the data were uploaded for review by the study team. There was no in-person contact between participants and study personnel.

All monitor data were initially scanned and classified by iRhythm. Summary data and tracings were independently reviewed and interpreted by a board-certified cardiac electrophysiologist (TD). Supraventricular tachycardia (SVT) and ventricular tachycardia (VT) were classified as non-sustained if the arrhythmia continued for $>3$ beats but less than $30 \mathrm{~s}$ in duration, while an arrhythmia that persisted for $\geq 30 \mathrm{~s}$ was considered sustained. A participant-triggered event was considered to correlate with a cardiac rhythm disturbance if any rhythm other than sinus was observed during the $45 \mathrm{~s}$ prior to the trigger. We hypothesised that the mean sinus rate would decrease over the duration of monitoring as individuals recovered from their infection. To asses for changes in mean heart rate and to minimise confounding due to differences in participant activity, the mean heart rate over a 20 min period was assessed at 02:00 and compared between the first and last days of monitoring.

iRhythm provided ambulatory monitoring devices but played no role in the design, statistical analysis or presentation of the study.

\section{Statistical analysis}

Continuous variables are presented as means $\pm \mathrm{SD}$ or medians and IQR. Normally distributed continuous variables were compared using t-tests, non-normally distributed continuous variables with the Wilcoxon rank-sum test and categorical variables with the $\chi^{2}$ test. Stata V.12 (StataCorp, College Station, Texas) was used for statistical analyses. A two-tailed alpha of 0.05 was considered statistically significant.

\section{RESULTS}

Between 8 May 2020 and 21 December 2020, 51 participants with a confirmed positive COVID-19 test underwent 14-day ambulatory electrocardiographic monitoring. The median interval between COVID-19 testing and the start of monitoring was 75 (IQR 34-126, minimum 8) days. In general, participants were relatively young and without significant comorbid conditions (table 1). No participants had a history of coronary artery disease or heart failure. Six individuals (12\%) reported a history of palpitations prior to COVID-19 infection, although none carried a cardiac arrhythmia diagnosis. While all participants suffered from at least one COVID-related symptom at the time of diagnosis, relatively few required hospitalisation or treatment in an intensive care unit (ICU) (table 1). No participant received oxygen supplementation outside of the hospital setting.

At the time of study enrolment, $23(45 \%)$ participants continued to experience COVID-19 related symptoms and 17 (33\%) complained of palpitations since testing positive. During the monitoring period, no individuals were receiving active treatment with antiviral or other disease-specific pharmacologic therapy for COVID-19. 


\begin{tabular}{|c|c|}
\hline & $(n=51)$ \\
\hline Age, years, mean $\pm S D$ & $42 \pm 11$ \\
\hline Female gender, $\mathrm{n}(\%)$ & $33(65)$ \\
\hline \multicolumn{2}{|l|}{ Race/ethnicity } \\
\hline White, n (\%) & $36(70)$ \\
\hline African American, $\mathrm{n}(\%)$ & $1(2)$ \\
\hline Asian/Pacific Islander, $\mathrm{n}(\%)$ & $7(14)$ \\
\hline Other, n (\%) & $7(14)$ \\
\hline Bachelor's degree or higher, $\mathrm{n}(\%)$ & $44(86)$ \\
\hline Active inhaled tobacco/drug use, $n(\%)$ & $7(14)$ \\
\hline \multicolumn{2}{|l|}{ Medical history } \\
\hline Hypertension, n (\%) & $5(10)$ \\
\hline Diabetes, n (\%) & $2(4)$ \\
\hline HIV, n (\%) & $2(4)$ \\
\hline \multicolumn{2}{|l|}{ COVID-19 history } \\
\hline Any symptom, n (\%) & $51(100)$ \\
\hline \multicolumn{2}{|l|}{ Symptom severity $†$} \\
\hline Mild & $7(14)$ \\
\hline Moderate & $19(37)$ \\
\hline Severe & $25(49)$ \\
\hline Hospitalisation, n (\%) & $4(8)$ \\
\hline ICU treatment, $\mathrm{n}(\%)$ & $1(2)$ \\
\hline Mechanical ventilation, $\mathrm{n}(\%)$ & 0 \\
\hline
\end{tabular}

${ }^{*}$ Cough, pharyngitis, fever, dyspnoea and/or anosmia. $\dagger$ Mild=no limitation, moderate=slight limitation and severe=significant limitation in daily activities.

ICU, intensive care unit.

Median monitoring duration was 13.2 (IQR 10.5-13.8) days (table 2). The predominant rhythm was sinus in all cases. The mean sinus rate was $79 \pm 10$ (minimum mean 51, maximum mean 99) beats per minute. One participant demonstrated an isolated supraventricular ectopic burden $>1 \%$ (burden 2.8\%) and a second participant demonstrated an isolated ventricular ectopic burden $>1 \%$ (burden $15.4 \%$ with a single, dominant QRS morphology that accounted for $95 \%$ of ventricular ectopy). All remaining individuals ( $\mathrm{n}=49$ (96\%), 95\% CI $87 \%$ to $99.5 \%)$ had both a supraventricular and ventricular ectopic burden of $<1 \%$.

The participant with the $2.8 \%$ supraventricular ectopic burden was a 49-year-old woman with a history of hypertension and diabetes. She described her peak COVID-19 symptoms as severe but was asymptomatic at the time of enrolment. She was not hospitalised for her infection. She did not complain of palpitations either before or after her COVID-19 diagnosis. Her monitor was started 189 days after her positive test result. Among five participant-triggered events seen on her monitor, four $(80 \%)$ corresponded to sinus with supraventricular ectopy. The participant with the $15.4 \%$ ventricular ectopic burden was a 68 -year-old man with a history of hypertension and no prior palpitations. He
Table 2 Summary of 14-day ambulatory monitor results

\begin{tabular}{|c|c|}
\hline Monitor duration, days, median (IQR) & $13.2(10.5-13.8)$ \\
\hline Mean sinus rate, beats/min, mean $\pm S D$ & $79 \pm 10$ \\
\hline $\begin{array}{l}\text { Participants with at least one symptomatic } \\
\text { trigger, } \mathrm{n}(\%)\end{array}$ & $47(92)$ \\
\hline \multicolumn{2}{|l|}{ Supraventricular arrhythmias* } \\
\hline Supraventricular ectopic burden $>1 \%, \mathrm{n}(\%)$ & $1(2)$ \\
\hline Non-sustained SVT episodes, n (\%) & $22(43)$ \\
\hline Sustained SVT episodes, n (\%) & 0 \\
\hline Atrial fibrillation or atrial flutter, $\mathrm{n}(\%)$ & 0 \\
\hline \multicolumn{2}{|l|}{ Ventricular arrhythmias* } \\
\hline Ventricular ectopic burden>1\%, n (\%) & $1(2)$ \\
\hline Non-sustained VT episodes, n (\%) & $4(8)$ \\
\hline Sustained VT episodes, n (\%) & 0 \\
\hline \multicolumn{2}{|l|}{ Participant-triggered events $(\mathrm{n}=558)$} \\
\hline Ectopy or arrhythmia identified, n (\%) & $122(22)$ \\
\hline Sinus tachycardia, $\mathrm{n}(\%)$ & $113(20)$ \\
\hline Sinus rhythm, $\mathrm{n}(\%) \dagger$ & $323(58)$ \\
\hline
\end{tabular}

A total of 51 monitors were analysed.

*Statistics refer to the number and proportion of individual participants with the corresponding arrhythmia finding. †Events without ectopy and a sinus rate $<100$ beats per minute. SVT, supraventricular tachycardia; VT, ventricular tachycardia.

was hospitalised for COVID-19 and was treated with remdesivir, but was without ongoing COVID-19 symptoms at the time of enrolment. His monitor was started 48 days after his positive test result. He developed new palpitations after his COVID-19 diagnosis. Among six participant-triggered events seen on his monitor, five (83\%) corresponded to sinus with ventricular ectopy. Importantly, this patient had evidence of frequent monomorphic ventricular ectopy on both a resting ECG and during a regadenoson stress test performed 1 month prior to his COVID-19 diagnosis. These Premature ventricular contractions localised to the superior mitral annulus and were present at a $25 \%$ burden on the resting ECG and at an $18 \%$ burden during stress testing.

A total of 22 participants (43\%) demonstrated nonsustained SVT. Among those with non-sustained SVT, the median number of episodes was 2 (IQR 1-10) and the median duration of the longest SVT episode per participant was 8 (IQR 5-18) beats. The longest overall episode of nonsustained SVT was 38 beats in duration. Four participants (8\%) demonstrated non-sustained VT; a single episode was observed in three individuals, while five episodes were seen in the remaining participant. The longest observed episode of non-sustained VT was eight beats in duration. All episodes were monomorphic in appearance. None of the non-sustained VT episodes were associated with participanttriggered events.

No atrial fibrillation or atrial flutter was identified. There were no sustained atrial or ventricular arrhythmias. Second degree Mobitz I (Wenckebach) AV block was noted in eight participants; none of these episodes were associated 


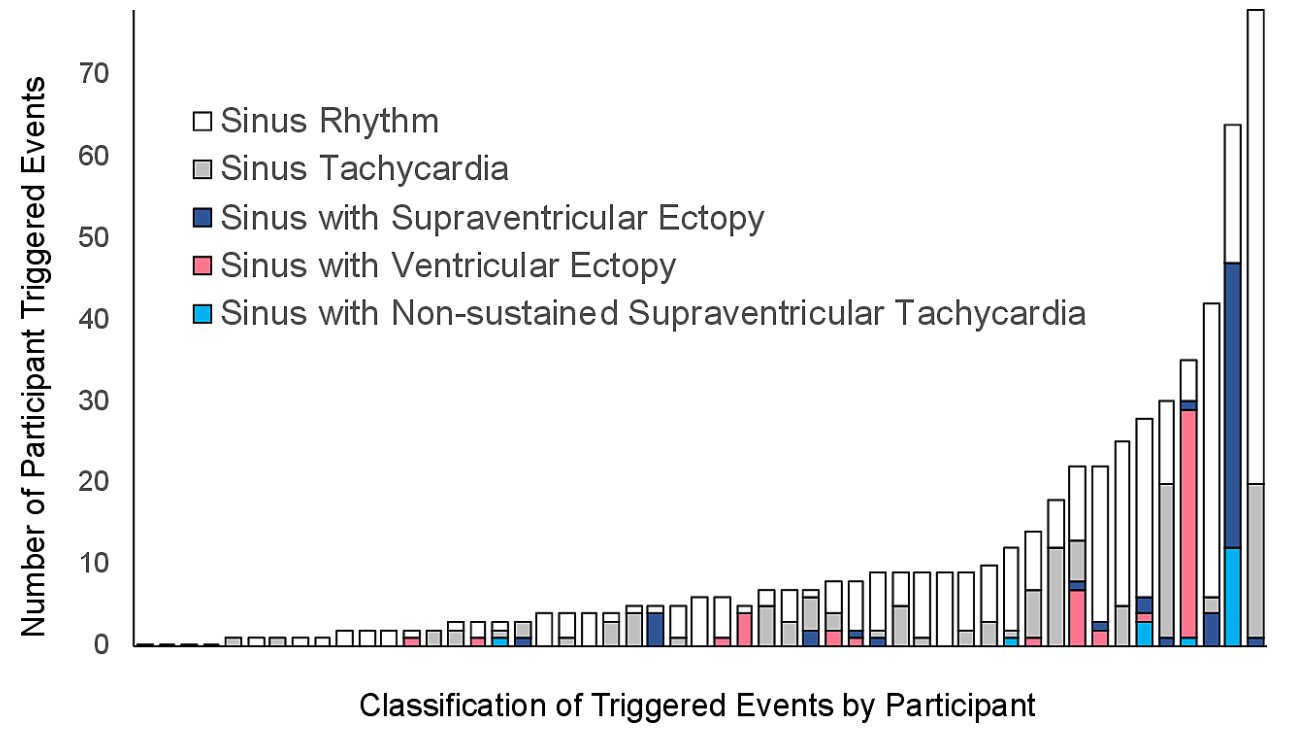

Figure 1 Number and classification of participant-triggered events during 14-day ambulatory electrocardiographic monitoring. None of the patient-triggered events were associated with non-sustained ventricular tachycardia. No sustained arrhythmias were observed.

with participant-triggered events. No instances of Mobitz II second-degree or third-degree AV block were identified.

A total of 47 participants (92\%) triggered the monitor at least once. There was a median 6 (IQR 3-12) triggers per individual. The vast majority of these triggers were for either sinus rhythm $(58 \%)$ or sinus tachycardia $(20 \%)$. Triggered events containing isolated supraventricular ectopy $(10 \%)$, isolated ventricular ectopy (9\%), or non-sustained SVT (3\%) were comparatively rare (figure 1 ). More than half $(55 \%)$ of participants with any trigger activation manifested only sinus rhythm or sinus tachycardia during symptoms.

There were no significant associations between measured clinical factors and a higher mean sinus rate. Specifically, neither time since COVID-19 diagnosis, peak COVID-19 symptom severity, the presence of ongoing COVID-19 symptoms, presence of palpitations since COVID-19 diagnosis nor history of COVID-19 hospitalisation predicted an elevated mean heart rate. The mean sinus rate at 02:00 increased between the first and last days of monitoring, although the difference in rate was small $(67 \mathrm{vs} 70 \mathrm{bpm})$ and was of borderline statistical significance $(\mathrm{p}=0.05)$. Participants who reported palpitations after their COVID-19 diagnosis at their baseline study visit triggered their monitor more frequently compared with those without palpitations (median 12 (IQR 5-30) triggers vs 4 (IQR 2-8), p=0.003). Otherwise, the number of participant-triggered events was not significantly associated with time since diagnosis, ongoing COVID-19 symptoms, infection symptom severity or history of hospitalisation.

\section{DISCUSSION}

In this prospective observational cohort study that enrolled ambulatory individuals with a recent and confirmed COVID-19 infection, we found a very low incidence of clinically significant cardiac arrhythmias using continuous 14-day electrocardiographic monitoring. Specifically, no individual in our cohort had documented atrial fibrillation, atrial flutter, sustained SVT, sustained VT or infranodal atrioventricular block. Furthermore, $96 \%$ of participants $(95 \%$ CI $87 \%$ to $99.5 \%$ ) had a low atrial and ventricular burden that would generally be considered within the normal range $(<1 \%) .{ }^{11}$ While most participants triggered their monitor for palpitation symptoms, nearly $80 \%$ of these triggers were for either sinus rhythm or sinus tachycardia.

Initial case series that enrolled critically ill COVID-19 inpatients early in the pandemic observed high rates of cardiac arrhythmias during hospitalisation, including a 17\% risk of overall (but not further classified) arrhythmia ${ }^{8}$ and a $6 \%$ risk of VT/fibrillation. ${ }^{4}$ Subsequent reports with more detailed arrhythmia classification have found a lower risk of arrhythmia among hospitalised patients. ${ }^{6} 12$ Importantly, these analyses identified markers of heightened infection severity, including ICU admission or an elevated troponin, as arrhythmia risk factors. Critical illness is a known risk factor for arrhythmia development independent of viral infection, ${ }^{13}$ and it is therefore possible that these arrhythmias are primarily a manifestation of patient acuity and are not specific to COVID-19 infection. However, palpitations are a common symptom during the convalescent stage of the illness, and prior studies have not rigorously evaluated whether discernable arrhythmias are present.

In our analysis, $1 / 3$ of participants complained of palpitations after their COVID-19 diagnosis and 92\% triggered their monitor for acute palpitation symptoms. Despite the reasonably high frequency of this symptom, the majority of triggered events corresponded to sinus rhythm/sinus tachycardia. Frequent, persistent tachycardia has been previously noted for up to 3 weeks after hospital discharge among individuals with SARS, despite resolution of fever and haemodynamic instability. ${ }^{14}$ This prior analysis was limited by absence of electrocardiographic rhythm determination. In our cohort, the mean sinus rate was within a normal 
range for all participants, did not correlate with time since COVID-19 diagnosis and did not decrease over the 14-day monitoring period. The remainder of participant-triggered events with an objective rhythm abnormality (22\%) did not demonstrate malignant findings and instead correlated with isolated ectopy or non-sustained SVT. Taken together, the observed low arrhythmia burden despite frequent palpitation symptoms suggests that clinically significant arrhythmias are uncommon among most individuals after COVID-19 infection.

Two individuals in our cohort demonstrated an arrhythmia burden $>1 \%$, including one participant with a $2.8 \%$ supraventricular ectopic burden and one with a $15.4 \%$ ventricular ectopic burden. Notably, the participant with frequent ventricular ectopy demonstrated this abnormality prior to his COVID-19 diagnosis despite reporting palpitations only after his infection, indicating his arrhythmia was not due to COVID-19. The mechanisms through which a COVID-19 infection may promote atrial or ventricular ectopy are not presently known. A recent cardiac MRI study found that a very high proportion of individuals with prior COVID-19 infection had evidence of cardiac involvement and active inflammation (78\% and 60\%, respectively). ${ }^{15}$ Importantly, the majority of participants in this prior study were never hospitalised and the time between COVID-19 diagnosis and MRI imaging was nearly identical to the period between diagnosis and electrocardiographic monitoring in the present investigation. While these imaging findings provide a potential mechanism to explain arrhythmogenesis, they also indicate that there is a large discrepancy between the prevalence of abnormal imaging findings and demonstrable arrhythmias in the convalescence phase after COVID-19 infection.

Our study has several limitations that should be recognised. We did not enrol COVID-19-negative adults as controls. However, the overall arrhythmia burden in our study was very low and we believe it is unlikely that a comparator group with similar demographics would have a clinically significant reduction in observed burden. Our cohort was comprised of relatively young and healthy self-selected individuals who generally had a mild clinical course. It is possible that COVID-19 infection is more arrhythmogenic among older individuals with more comorbidities or in other types of individuals less likely to volunteer for our study. On the other hand, the low expected prevalence of arrhythmias in our sample makes it an ideal population within which to study the association between COVID-19 and arrhythmias due to the expected low ambient level of arrhythmias in this population. Furthermore, COVID-19 incidence is highest and rising most rapidly among individuals of age 18-44 years. ${ }^{16} 17$ Participants with symptoms potentially due to an arrhythmia may have been more inclined to volunteer for our study, although this would be expected to enhance detection of rhythm abnormalities, if present. Such bias therefore does not diminish our central finding of a low arrhythmia burden in this patient population. The fairly broad range of time from COVID-19 diagnosis to rhythm monitoring may have reduced our ability to reproducibly detect infection-associated arrhythmias, although no patient in our cohort had a serious arrhythmia and time since COVID-19 diagnosis was neither predictive of mean sinus rate nor number of patient-triggered events. We also acknowledge that our sample was relatively small; with only 51 participants we do not have the statistical power to detect a small increase in arrhythmias in this population. Finally, we did not perform systematic cardiac imaging in our cohort.

In this prospective cohort study, we did not find evidence of malignant or sustained arrhythmias in outpatients after a positive COVID-19 diagnosis. While palpitations were common, these symptoms frequently corresponded to sinus rhythm/sinus tachycardia or nonmalignant arrhythmias such as isolated ectopy or nonsustained SVT. Although these findings cannot exclude the possibility of serious arrhythmia in selected individuals, they do not support a strong or widespread proarrhythmic effect of COVID-19 infection after resolution of acute illness.

Contributors TD and GM designed the study, drafted the manuscript and are responsible for the overall content as guarantor and thereby accept full responsibility for the finished work and the conduct of the study, including access to the data. TD performed all statistical analyses. All authors provided substantial contributions to data interpretation and critical revisions and approved the final manuscript.

Funding This work was made possible by the NIH/NIBIB grant number 3U2CEB021881-05S1 (GM, MP, JE0).

Competing interests UK discloses full-time salary support and significant stock ownership in Element Science, Inc. and significant stock ownership in iRhythm Technologies, Inc.

Patient consent for publication Not applicable.

Ethics approval This study involves human participants and was approved by the UCSF Institutional Review Board, IRB Number: 13-11663. Participants gave electronic consent to participate in the study before taking part.

Provenance and peer review Not commissioned; externally peer reviewed.

Data availability statement Data are available upon reasonable request.

Open access This is an open access article distributed in accordance with the Creative Commons Attribution Non Commercial (CC BY-NC 4.0) license, which permits others to distribute, remix, adapt, build upon this work non-commercially, and license their derivative works on different terms, provided the original work is properly cited, appropriate credit is given, any changes made indicated, and the use is non-commercial. See: http://creativecommons.org/licenses/by-nc/4.0/.

ORCID IDs

Thomas A Dewland http://orcid.org/0000-0001-6069-7135

David Wen http://orcid.org/0000-0003-1986-2164

\section{REFERENCES}

1 Escher F, Pietsch H, Aleshcheva G, et al. Detection of viral SARSCoV-2 genomes and histopathological changes in endomyocardial biopsies. ESC Heart Fail 2020;7:2440-7.

2 Nishiga M, Wang DW, Han Y, et al. COVID-19 and cardiovascular disease: from basic mechanisms to clinical perspectives. Nat Rev Cardiol 2020;17:543-58.

3 Shi S, Qin M, Shen B, et al. Association of cardiac injury with mortality in hospitalized patients with COVID-19 in Wuhan, China. JAMA Cardiol 2020;5:802-10.

4 Guo T, Fan Y, Chen M, et al. Cardiovascular implications of fatal outcomes of patients with coronavirus disease 2019 (COVID-19). JAMA Cardiol 2020;5:811-8. 
5 Cho JH, Namazi A, Shelton R, et al. Cardiac arrhythmias in hospitalized patients with COVID-19: a prospective observational study in the western United States. PLoS One 2020;15:e0244533.

6 Bhatla A, Mayer MM, Adusumalli S, et al. COVID-19 and cardiac arrhythmias. Heart Rhythm 2020;17:1439-44.

7 Linschoten M, Peters S, van Smeden M, et al. Cardiac complications in patients hospitalised with COVID-19. Eur Heart $J$ Acute Cardiovasc Care 2020;9:817-23.

8 Wang D, Hu B, Hu C, et al. Clinical characteristics of 138 hospitalized patients with 2019 novel coronavirus-infected pneumonia in Wuhan, China. JAMA 2020;323:1061-9.

9 Carvalho-Schneider C, Laurent E, Lemaignen A, et al. Follow-Up of adults with noncritical COVID-19 two months after symptom onset. Clin Microbiol Infect 2021;27:258-63.

10 Liang L, Yang B, Jiang N, et al. Three-Month follow-up study of survivors of coronavirus disease 2019 after discharge. J Korean Med Sci 2020;35:e418.
11 Marcus GM, Marcus Gregory M. Evaluation and management of premature ventricular complexes. Circulation 2020;141:1404-18.

12 Colon CM, Barrios JG, Chiles JW, et al. Atrial arrhythmias in COVID-19 patients. JACC Clin Electrophysiol 2020;6:1189-90.

13 Annane D, Sébille V, Duboc D, et al. Incidence and prognosis of sustained arrhythmias in critically ill patients. Am J Respir Crit Care Med 2008;178:20-5.

14 Yu C-M, Wong RS-M, Wu EB, et al. Cardiovascular complications of severe acute respiratory syndrome. Postgrad Med J 2006;82:140-4.

15 Puntmann VO, Carerj ML, Wieters I, et al. Outcomes of cardiovascular magnetic resonance imaging in patients recently recovered from coronavirus disease 2019 (COVID-19). JAMA Cardiol 2020;5:1265.

16 Venkatesan P. The changing demographics of COVID-19. Lancet Respir Med 2020;8:e95.

17 COVID-19 Stats: COVID-19 Incidence, ${ }^{*}$ by Age Group $\dagger$ - United States, March 1-November 14, 2020§. MMWR Morb Mortal Wkly $\operatorname{Rep~2021;69:1664.~}$ 